\title{
On the long-distance charge transport in DNA-like macromolecules
}

\author{
D. Chevizovich ${ }^{1}$, A. V. Chizhov ${ }^{2,3}$, Z. Ivić ${ }^{1}$, A. A. Reshetnyak ${ }^{4}$ \\ ${ }^{1}$ University of Belgrade, Vinča Institute of Nuclear Sciences, \\ Laboratory for Theoretical and Condensed Matter Physics, \\ P.O. BOX 522, Belgrade, 11001, Serbia \\ ${ }^{2}$ Laboratory of Radiation Biology, Joint Institute for Nuclear Research, \\ Joliot-Curie, 6, Dubna, 141980, Russia \\ ${ }^{3}$ Dubna State University, Universitetskaya, 19, Dubna, 141980, Russia \\ ${ }^{4}$ Institute of Strength Physics and Materials Science SB RAS, \\ Academichesky ave., 2/4, Tomsk, 634021, Russia \\ cevizd@vinca.rs
}

PACS 87.15.hj, 05.60.Gg, 05.45.Yv, 87.10.-e,

DOI 10.17586/2220-8054-2021-12-1-32-41

\begin{abstract}
In this paper, we investigate the possibility of stable migration of charge carriers over long distances in DNA-like macromolecular structures in the form of an adiabatic soliton and derive the conditions for the formation of solitons. We find two types of soliton solutions: symmetric and antisymmetric. Comparing the energy of both types of soliton solutions with the energy of free extra charge, we find the region of the system parameters in which the soliton states are more energetically favorable than the states of quasi-free charges. At the same time, which of the two mentioned soliton solutions corresponds to an energetically favorable state depends on the ratio of the energy parameters of the molecular structure.
\end{abstract}

Keywords: charge migration, DNA, soliton, adiabatic polaron, interchain coupling.

Received: 22 December 2020

Revised: 25 December 2020

\section{Introduction}

Deoxyribonucleic acid (DNA) is a stable macromolecular structure that carries the genetic information of living organisms. This molecule stores significant amounts of information that plays a key role in the functioning of a living cell, such as that related to the synthesis of vital proteins, for example. The primary structure of DNA consists of two polynucleotide chains, each of which is made up of a series of nucleotides linked to each other via phosphate $\mathrm{PO}_{4}$ and hydroxyl $\mathrm{OH}$ groups, which are located on the sugar components. Specific nucleotide residues are purine and pyrimidine bases: adenine (A), guanine $(\mathrm{G})$, thymine $(\mathrm{T})$ and cytosine $(\mathrm{C})$. The bases of one strand are linked to the complementary base belonging to other strand by a network of hydrogen bonds: adenine is linked to thymine (A-T) by double hydrogen bond, and guanine is linked to cytosine $(\mathrm{G}-\mathrm{C})$ by a triple hydrogen bond. In this way, a doublestranded DNA macromolecule is formed. The secondary structure of DNA is the double helix. For the biological functionality of DNA the primary and secondary structures are responsible [1,2].

The appearance and migration of an extra charge in DNA can have a major impact on the functioning of this molecule [1,3-7]. For example, one source of DNA damage can be the oxidative stress process and UV irradiation, when an electron is removed from nucleotide and an extra positive charge (hole) is created. A major target for oxidants is guanine, since it has the lowest ionization potential compared to other DNA bases. The oxidative stress
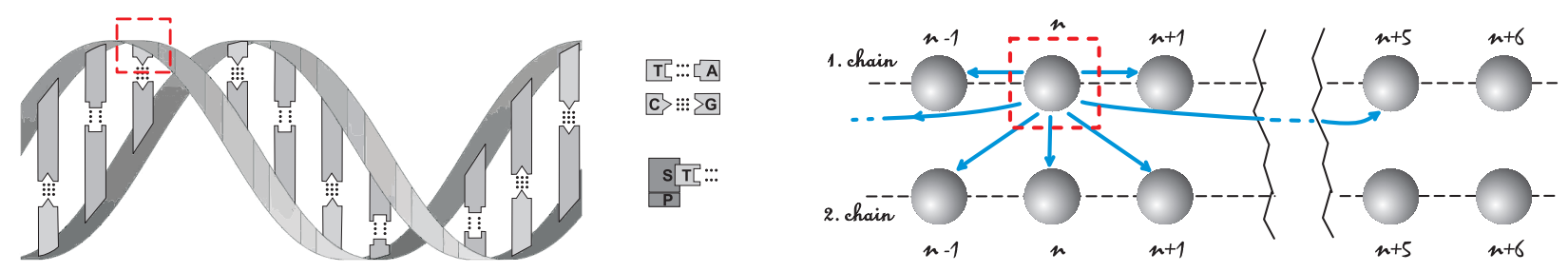

FIG. 1. Left panel: Schematic structure of the double helix DNA macromolecule. The backbone of the DNA strand is made of alternating phosphate and sugar groups. Right panel: Simplified structure of DNA-like macromolecule. Nucleotide bases are presented by spheres, regularly distributed along the strains. The most probable directions of charge migration from a given nucleotide (enabled by the overlaping of electron $\pi$-orbitals between neighboring nucleotides) are marked with arrows. 
on guanine can reduce fidelity in the replication of DNA molecule and, consequently, such a process is the source of genomic mutations [2-4]. Further, the overlap of electronic $\pi$-orbitals of neighboring nucleotide groups will lead to delocalization of the charge along the chain and its capture by a neighboring nucleotide, having a lower oxidation potential. Because guanine has the lowest oxidation potential, the migration of the extra charge will occur along the guanines and all other nucleotides will represent potential barriers for its movement. Due to the fact that multiguanine sequences have a lower ionization potential than a single guanine, the formed positive charge must migrate to guanine clusters, sometimes over long distances $[1,3,4]$. The mentioned mechanism can cause the appearance of the mutation not only at the place where the oxidative stress process occurred, but at any place along the DNA chain. Compared to the migration of positive charge that occurs in the process of the oxidative stress on guanine, much less is known about electron migration via base radical anions. The bases that are most easily reduced are $\mathrm{T}$ and $\mathrm{C}$, whose reduction potentials are very similar. Therefore, it was assumed that the migration mechanism might involve all base pairs as charge carriers [3]. As a consequence, the question arises about the mechanism of the stable long range charge migration in DNA molecule, as well as in molecules that have a structure similar to DNA. Two mechanisms of charge migration in DNA are most often mentioned in the literature. The first is based on a tunnelling effect, but this mechanism cannot explain the long-distance charge migration. The second mechanism is based on the $\pi$-orbital overlap between neighboring nucleotide groups which leads to charge delocalization along the chain and its capture by the neighboring nucleotide $[1,3,4,8,9]$. It leads to charge migration as a series of successive jumps from one nucleotide base to the neighboring one. This mechanism corresponds to the transport of the Holstein nonadiabatic polaron, which, in principle, is able to explain the stable long-range charge transport [10,11].

To form such a quasiparticle, the macromolecule must satisfy certain conditions for basic energy parameters of a structure: phonon energy, energy of the extra charge transitions from one to the adjancent structure elements (which is related to the values of the transfer integrals for the extra charge), and the strength of interaction of the extra charge with the phonon subsystem (which is related to the value of the particle-phonon coupling constant $\chi$ and determines the polaron binding energy $E_{b}$ ) $[7,10,12-16]$. Investigations devoted to the determination of the values of these parameters in DNA as well as in other biological macromolecules is still ongoing, but, according to various results, estimated values of these parameters (especially those concerning $\chi$ ) explore the whole regime ranging from weak to strong coupling limit $[17,18]$. Therefore, in addition to the hopping mechanism of the quasi-free and non-adiabatic (small) polaron migration, it is necessary to consider other possibilities.

In this paper, we consider the possibility that an induced charged particle (due to interaction with phonons of the structure) forms an adiabatic polaron (soliton). For this purpose, a simple molecular structure in the form of a double helix with identical structural elements is considered, in which the difference between different nucleotide residues is ignored and it is assumed that each of them can be a target for an extra charge with the same efficiency.

Here, we assume that the $\pi$-orbital overlap can occur not only between two adjacent structural elements belonging to the same chain (which causes migration along the same chain), but also between two structural elements belonging to different chains. The assumptions made allow us to obtain a simple analytical model that can give a much clearer picture of the charge migration process in DNA-like molecules, but can also serve as a starting point for more accurate numerical calculations.

\section{The model}

We consider an extra charge (exciton in general) in a structure consisting of the two coupled identical macromolecular chains, each of which is composed of $N \gg 1$ structural elements (molecular groups). The physics of charge migration in such a structure is usually described in the framework of common tight binding model, modified here in order to incorporate the interchain coupling of charge excitations on different chains. The helical structure is taken into account in such a way that we allow delocalization of the charge from the $n$-th node (where it is located) to the node that is close to its current position due to the helical form of the macromolecule ( $n \pm \delta$ node). For DNA macromolecule, $\delta=5$ (see Fig. 1). As a consequence, we allow the charge to be able to migrate from $n$-th to $n \pm 1$ node as well as from $n$-th to $n \pm \delta$-node along the same chain. In addition, due to the spatial proximity, we allow the charge to migrate from $n$-th node of one chain to $n$-th and $n \pm 1$-th nodes placed on another chain. We take into account the charge coupling with acoustic phonon modes. Only local exciton-phonon coupling is considered. Under these conditions, the model Hamiltonian has the form:

$$
\hat{H}=\hat{H}_{e}+\hat{H}_{p h}+\hat{H}_{i n t}
$$


where the exciton Hamiltonian is:

$$
\begin{aligned}
\hat{H}_{e} & =\mathcal{E}_{0} \sum_{n, j} \hat{a}_{n, j}^{\dagger} \hat{a}_{n, j}-J_{1} \sum_{n, j} \hat{a}_{n, j}^{\dagger}\left(\hat{a}_{n+1, j}+\hat{a}_{n-1, j}\right) \\
& +J_{\delta} \sum_{n, j} \hat{a}_{n, j}^{\dagger}\left(\hat{a}_{n+\delta, j}+\hat{a}_{n-\delta, j}\right)+L_{1} \sum_{n, j} \hat{a}_{n, j}^{\dagger} \hat{a}_{n, 3-j} \\
& +L_{2} \sum_{n, j} \hat{a}_{n, j}^{\dagger}\left(\hat{a}_{n+1,3-j}+\hat{a}_{n-1,3-j}\right),
\end{aligned}
$$

the phonon Hamiltonian is:

$$
\hat{H}_{p h}=\sum_{q, j} \hbar \omega_{q} \hat{b}_{q, j}^{\dagger} \hat{b}_{q, j}
$$

and, finally, the Hamiltonian of exciton-phonon interaction is:

$$
\hat{H}_{i n t}=\frac{1}{\sqrt{N}} \sum_{n, q, j} F_{q} \mathrm{e}^{i q n R_{0}} \hat{a}_{n, j}^{\dagger} \hat{a}_{n, j}\left(\hat{b}_{q, j}+\hat{b}_{-q, j}^{\dagger}\right) .
$$

Here $n$ labels the lattice sites along the strands, and it takes the values from $-N / 2$ to $N / 2$. Index $j$ enumerates molecular strands $(j=1,2), R_{0}$ is the distance between two adjacent structural elements of the same chain, operators $\hat{a}_{n, j}^{\dagger}\left(\hat{a}_{n, j}\right)$ correspond to the exciton creation (annihilation) operators on $n$-th site of the $j$-th strand, and $\hat{b}_{q, j}^{\dagger}\left(\hat{b}_{q, j}\right)$ correspond to the phonon creation (annihilation) operators of the $q$-th phonon mode ( $q$ is the phonon wave vector) on the $j$-th strand. $\mathcal{E}_{0}$ is the energy of charge excitation on the corresponding $(n, j)$ molecule, $J_{1}$ is the transfer integral between the neighboring molecules on the same strand (we adopt that $J_{1}>0$ ), while $J_{\delta}$ is the transfer integral between sites $n$ and $n \pm \delta$ on the same strand, which originates from the helical structure of the macromolecule. Finally, $L_{1}$ and $L_{2}$ are the transfer integrals between the nearest $((n, j) \leftrightarrow(n, 3-j))$ and the first neighboring $((n, j) \leftrightarrow(n \pm 1,3-j))$ molecules on different chains. In the case of Peierls dielectrics, it is commonly adopted that $L_{1}<0$, but in some substances it may be of the opposite sign or it may alternate along a chain [19]! Here, we adopt that $J_{1}, J_{\delta}, L_{1}$, and $L_{2}$ have the same values along the structure. We addopt that $J_{1}$ is positive, while other transfer integrals can be either positive or negative.

The interaction of the charge with the phonon subsystem is determined through the exciton-phonon coupling parameter $F_{q}$. The form of this parameter depends on the phonon's nature. In what follows, we will assume that the mechanical oscillations of both chains are mutually independent, which can be justified by the fact that the interaction energies of adjacent two structural elements of different molecular chains are significantly less than the interaction energies of two adjacent structural elements belonging to the same chain. In addition, we suppose that the electron interacts with acoustic phonon modes only [20-22]. For that reason, the exciton-phonon coupling parameter has the form: $F_{q}=2 i \chi \sqrt{\hbar / 2 M \omega_{q}} \sin \left(q R_{0}\right)$, where $\omega_{q}=\omega_{0} \sin q R_{0} / 2$ is the phonon frequency, and $\omega_{0}=2 \sqrt{\kappa / M}$ is the characteristic phonon energy. Here, parameter $\chi$ is the strength of exciton-phonon interaction, $\kappa$ is the stiffness of the particular chain, and $M$ is the mass of the molecular group at the chain site $n$. Finally, we adopt the linear dispersion law for phonons: $\omega_{q}=c|q|$, where $c=\omega_{0} R_{0} / 2$.

In order to discuss the stable long-distance charge migration along the DNA structure, we suppose that, due to the charge-phonon interaction, the extra charge forms a soliton-like large polaron. The nature of the formed polaron quasiparticle, in the case of macromolecular structures, depends on the values of the basic energy parameters, such as the transfer integrals $J_{1}$ of the charge transfer between the nearest neighboring molecular group along the same chain (here, we suppose that the interchain transfer integral $L$ is lower than $J_{1}$ and, consequently, its influence on the polaron formation is neglected), so called the polaron binding energy $E_{b}=1 / N \sum_{q}\left|F_{q}\right|^{2} / \hbar \omega_{q}=8 \chi^{2} / M \omega_{0}^{2}$, and the characteristic phonon energy $\hbar \omega_{0}$. The concept of a large polaron is applicable to structures that satisfy the following condition $[7,12,13,23,24]$ :

$$
2 J_{1} \gg E_{b} \gg \hbar \omega_{0} .
$$

Here, the adiabatic condition $\left(2 J_{1} \gg \hbar \omega_{0}\right)$ ensures that the fluctuations of charge and macromolecular subsystems are uncorrelated, and they can be neglected [7,23,24]. As a consequence, the mean values of the products of the charge and phonon operators may be factorized, and theoretical treatment can be carried out within the semiclassical approximation, for example, in the framework of the Pekar variational anzats [12,23,24]. Physically, this means that the deformation of the lattice formed due to the interaction of excitons with the phonon subsystem is slow compared to the exciton subsystem, and it can not follow the quasiparticle motion (deformation of the lattice forms a "frozen" structure). The strong coupling condition $E_{b} \gg \hbar \omega_{0}$ ensures that the potential well that appears due to the lattice distortion (and which captures the extra charge) is deep enough to prevent the destruction of the formed polaron state 
(it provides the polaron stability). On the other hand, this coupling should not be very strong in order to allow the polaron to span a large number of lattice sites, when continuum approximation is applicable. Such a condition is satisfied in the case when $2 J_{1} \gg E_{b}[12,13]$. On the basic of existing data, it seems that the mentioned criteria are satisfied for many biological macromolecular structures, including DNA. As usual, in most conjugated polymers, the typical values of the phonon energies are in the range $0.12 \mathrm{eV}$ in polyacene molecule, $0.2 \mathrm{eV}$ in double-strand polyacene, and $17 \mathrm{meV}$ in alpha-helix structure. In the case of DNA, phonon energies are about $\hbar \omega_{0}=1 \mathrm{meV}$ [25]. Typical values for the intersite transfer integral are approximately $2.5 \mathrm{eV}$ in conjugated polymers, while in alpha-helix its value is estimated on the order of few eV. According to different calculations, the values of the electron and hole transfer integrals in DNA are about $J_{1} \approx 0.1 \mathrm{eV}[18,26]$. The values for the charge-phonon coupling parameter lie in the entire range from the weak to the strong charge-phonon coupling limit. They are estimated in several eV/A: for polyacene $\chi \approx 4.1 \mathrm{eV} / \mathrm{A}$, and for DNA $\chi$ ranges from $0.3 \mathrm{eV} / \mathrm{A}$ to $2.3 \mathrm{eV} / \mathrm{A}$ [17].

In order to describe the process of ST in structures consisting of two or more chains, it is necessary to introduce additional parameters which will take into account additional interactions between the chains and other interactions arising from additional geometric properties of the structure (helical geometry, for example): $p_{1}=L_{1} / J_{1}, p_{2}=$ $L_{2} / J_{1}$, and finally, $p_{\delta}=J_{\delta} / J_{1}$.

To study the dynamics of our system, we use the simple time dependent extension of the Pekar variational ansatz, that allows the simple factorization of charge and phonon variables [23, 24, 27, 28]:

$$
\left.|\psi\rangle=\sum_{n, j} \psi_{n, j}(t) \hat{a}_{n, j}^{\dagger}|0\rangle_{e} \otimes \beta(t)\right\rangle,
$$

where $|\beta(t)\rangle=\Pi_{q, j}\left|\beta_{q, j}(t)\right\rangle$ is the multimode phonon coherent state, and $\left|\beta_{q, j}(t)\right\rangle$ is the eigenfunction of the phonon annihilation operator: $\hat{b}_{q, j}(t)\left|\beta_{q, j}(t)\right\rangle=\beta_{q, j}(t)\left|\beta_{q, j}(t)\right\rangle$. Due to the conservation of the probability for the quasiparticle, exciton amplitudes are normalized. So that:

$$
\sum_{n, j}\left|\psi_{n, j}(t)\right|^{2}=1
$$

The functions $\psi_{n, j}(t)$ and $\beta_{q, j}(t)$ are treated as dynamical variables. In order to find exciton and phonon amplitudes, as a first step we find the explicit form of the functional $\mathcal{H}=\langle\psi|\hat{H}| \psi\rangle$ :

$$
\begin{aligned}
\mathcal{H} & =\mathcal{E}_{0} \sum_{n, j} \psi_{n, j}^{*} \psi_{n, j}-J_{1} \sum_{n, j} \psi_{n, j}^{*}\left(\psi_{n+1, j}+\psi_{n-1, j}\right) \\
& +J_{\delta} \sum_{n, j} \psi_{n, j}^{*}\left(\psi_{n+\delta, j}+\psi_{n-\delta, j}\right)+\sum_{q, j} \hbar \omega_{q} \beta_{q, j}^{*} \beta_{q, j} \\
& +L_{1} \sum_{n, j} \psi_{n, j}^{*} \psi_{n, 3-j}+L_{2} \sum_{n, j} \psi_{n, j}^{*}\left(\psi_{n+1,3-j}+\psi_{n-1,3-j}\right) \\
& +\frac{1}{\sqrt{N}} \sum_{n, q, j} F_{q} \mathrm{e}^{i q n R_{0}} \psi_{n, j}^{*} \psi_{n, j}\left(\beta_{q, j}+\beta_{-q, j}^{*}\right) .
\end{aligned}
$$

Equations of motion for dynamical variables $\psi_{n, j}(t)$ and $\beta_{q, j}(t)$ are:

$$
i \hbar \dot{\psi}_{n, j}=\frac{\partial \mathcal{H}}{\partial \psi_{n, j}^{*}}, \quad i \hbar \dot{\beta}_{q, j}=\frac{\partial \mathcal{H}}{\partial \beta_{q, j}^{*}} .
$$

In the continuum limit:

$$
\psi_{n, j}(t) \rightarrow \psi_{j}(x, t), \quad n R_{0} \rightarrow x, \quad \sum_{n} \rightarrow \int \frac{d x}{R_{0}}
$$

we have:

$$
\begin{aligned}
i \hbar \dot{\psi}_{j}(x, t) & =\left(\mathcal{E}_{0}-2 J_{1}+2 J_{\delta}\right) \psi_{j}(x, t)-\left(J_{1}-\delta^{2} J_{\delta}\right) R_{0}^{2} \frac{\partial^{2} \psi_{j}(x, t)}{\partial x^{2}} \\
& +L_{2} R_{0}^{2} \frac{\partial^{2} \psi_{3-j}(x, t)}{\partial x^{2}}+\left(L_{1}+2 L_{2}\right) \psi_{3-j}(x, t) \\
& +\frac{1}{\sqrt{N}} \sum_{q} F_{q} \mathrm{e}^{i q x} \psi_{j}(x, t)\left(\beta_{q, j}(t)+\beta_{-q, j}^{*}(t)\right),
\end{aligned}
$$


and the equation of motion for the phonon amplitudes are:

$$
i \hbar \dot{\beta}_{q, j}(t)=\hbar \omega_{q} \beta_{q, j}(t)+\frac{F_{-q}}{\sqrt{N}} \int_{-\infty}^{+\infty} \frac{d x}{R_{0}} \mathrm{e}^{-i q x}\left|\psi_{j}(x, t)\right|^{2} .
$$

In the obtained system of equations (9) and (10) we have coupled charge and vibrational amplitudes. Since our goal is to analyze the properties of the extra charge, the first step we must take is to eliminate from the Eq. (9) the variables that describe the phonon subsystem. Because we investigate the extra charge propagation in the form of a stable "wave pulse" (soliton form), we assume that the charge probability densities depend on $t$ and $x$ through coordinate $\xi=x-v t:|\psi(x, t)|^{2}=|\psi(x-v t)|^{2}$, where $v$ is the soliton velocity. In this case, Eq. (10) becomes an ordinary differential equation of the first order, and it can be easily integrated. The general solution of this equation has the form: $\beta_{q, j}(t)=\beta_{q, j}^{\text {homog }}(0) \mathrm{e}^{-i \omega_{q} \cdot t}+\beta_{q, j}^{\text {coh }}(t)$. Here, the first term is the homogeneous solution corresponding to the incoherent part of lattice displacement coming from free lattice modes, and it can be disregarded in the adiabatic treatment $[7,29,30]$. Since we search for a stable stationary polaron solution, the main contribution to the polaron formation comes from the particular solution. The particular solution of the phonon equation of motion attains the form:

$$
\beta_{q, j}^{\mathrm{coh}}(t)=-\frac{1}{\sqrt{N}} \frac{F_{q, j}^{*}}{\hbar \omega_{q}} \frac{1}{1-\frac{q v}{\omega_{q}}} \int \frac{d x}{R_{0}} \mathrm{e}^{-i q x}\left|\psi_{j}(x, t)\right|^{2} .
$$

After substitution of the obtained phonon amplitudes (11) into (9), we obtain the system of the two coupled nonlinear Schrödinger equations (NSE) for polaron amplitudes $\psi_{1}(x, t)$ and $\psi_{2}(x, t)$ :

$$
\begin{aligned}
i \hbar \dot{\psi}_{j} & =-\left(J_{1}-\delta^{2} J_{\delta}\right) R_{0}^{2} \frac{\partial^{2} \psi_{j}}{\partial x^{2}}+L_{2} R_{0}^{2} \frac{\partial^{2} \psi_{3-j}}{\partial x^{2}} \\
& +\left(L_{1}+2 L_{2}\right) \psi_{3-j}-\frac{2 E_{b}}{1-v^{2} / c^{2}}\left|\psi_{j}\right|^{2} \psi_{j} .
\end{aligned}
$$

Physically irrelevant term $\left(\mathcal{E}_{0}-2 J_{1}+2 J_{\delta}\right) \psi_{j}(x, t)$ is absorbed by the transformation $\psi_{j}(x, t) \quad \rightarrow$ $\mathrm{e}^{\left(\mathcal{E}_{0}-2 J_{1}+2 J_{\delta}\right) \cdot t} \psi_{j}(x, t)$.

The resulting expression (12) depends on $J_{1}$ and $J_{\delta}$ only through the corrected transfer integral $J=J_{1}-\delta^{2} J_{\delta}$. Therefore, one can see that, in the framework of the presented model, $J_{\delta}$ corrects only the value of the exciton transfer integral $J_{1}$. Since the overlap of the $\pi$-orbitals between the $n$-th and $n \pm \delta$-th nodes in the chain is very small, the value of the corrected $J$ is rather close to $J_{1}$.

\section{Soliton solutions}

Thus, we have the system of two partial differential equations (12) for two unknown functions $\psi_{1}(x, t)$ and $\psi_{2}(x, t)$. Similar systems of equations have been studied in various problems, especially in biophysics and nonlinear optics [7,31-34]. Apart from two trivial cases, namely, $\psi_{1}(x, t) \neq 0, \psi_{2}(x, t)=0$ and $\psi_{1}(x, t)=0, \quad \psi_{2}(x, t) \neq 0$, the explicit solutions of such systems have not yet been fully examined, and they are generally unknown. Nevertheless, their properties may be examined by means of various approximate approaches. In this sense, the average profile approximation can be very useful as it allows analytical calculations, the accuracy of which has been demonstrated by comparison with numerical analysis [32-34]. The main idea of this approach is the assumption that the solutions of the coupled system of NSE are not substantially different from those of the uncoupled ones. Consequently, the stationary solutions of the above equations (12) can be sought in the form:

$$
\psi_{j}(x, t)=A_{j} \cdot \underbrace{\mathrm{e}^{i(k x-\Omega t)} \cdot \phi(x-v t)}_{\psi(x, t)}=A_{j} \psi(x, t) .
$$

Here, $A_{j}$ is the real amplitude of the soliton propagating along the molecular chain $j, k$ is the wave vector of the soliton carrier wave, $\Omega$ is the frequency of the carrier wave, $v$ is the soliton speed. The real function $\phi(x-v t)$ is the envelope of the extra charge wave function, which we looking for in the form of a wave pulse. This means that the initial conditions for envelope function are (in the "moving" coordinate frame, where $\xi=x-v t$ ):

$$
\begin{gathered}
\lim _{\xi \rightarrow \pm \infty} \phi(\xi)=\lim _{\xi \rightarrow \pm \infty} \frac{d \phi(\xi)}{d \xi}=0, \\
\lim _{\xi \rightarrow 0} \phi(\xi)=\phi_{0}, \quad \lim _{\xi \rightarrow 0} \frac{d \phi(\xi)}{d \xi}=0 .
\end{gathered}
$$


Since we accept the assumption that the DNA macromolecule is made up of two identical molecular strands, we can assume that the soliton wave has identical form on both macromolecular chains and it is $\psi(x, t)$ in (13). The normalization condition for the extra charge function in the continuum approximation becomes:

$$
\sum_{j} \int_{-\infty}^{+\infty} \frac{d x}{R_{0}} \psi_{j}^{*}(x, t) \psi_{j}(x, t)=\sum_{j} A_{j}^{2} \cdot \int_{-\infty}^{+\infty} \frac{d \xi}{R_{0}} \phi^{2}(\xi)=1
$$

If we additionally require that the envelope of the electronic wave function is normalized, the normalization condition becomes:

$$
\sum_{j} A_{j}^{2}=1, \quad \frac{1}{R_{0}} \int_{-\infty}^{+\infty} d \xi \phi^{2}(\xi)=1
$$

So, relations (12), (13), (14) and (15) are the mathematical framework for our study on the possible existence of solitons in DNA-like macromolecular structures. Let us analyze these equations and see under what conditions soliton solutions can occur. After substituting (13) into (12) and separating the real and imaginary parts of obtained equations, from the imaginary part we find the set of algebraic equations for amplitudes $A_{1}$ and $A_{2}$

$$
\begin{aligned}
& A_{1}\left(\hbar v-2 k R_{0}^{2} J\right)+A_{2} \cdot 2 k R_{0}^{2} L_{2}=0, \\
& A_{1} \cdot 2 k R_{0}^{2} L_{2}+A_{2}\left(\hbar v-2 k R_{0}^{2} J\right)=0 .
\end{aligned}
$$

The resulting system of algebraic equations has a nontrivial solution in the case when the determinant of the system is equal to zero. This condition yields two kinds of solutions, with the wave vector of the carrier wave in the form:

$$
k=\frac{m^{*} v}{\hbar}
$$

where the parameter $m^{*}$ can be considered as the soliton's effective mass. One of the solutions corresponds to the symmetric form for the amplitudes $A_{1}=A_{2}=1 / \sqrt{2}$, with the soliton's effective mass $m^{*}=\frac{\hbar^{2}}{2\left(J-L_{2}\right) R_{0}^{2}}$. Another solution corresponds to an antisymmetric representation for the amplitudes $A_{1}=-A_{2}=1 / \sqrt{2}$, with the soliton's effective mass $m^{*}=\frac{\hbar^{2}}{2\left(J+L_{2}\right) R_{0}^{2}}$.

The real part of Eq. (12) after substituting Eq. (13) into it determines the differential equation for the soliton envelope $\phi(x-v t)$, which, in the moving coordinate framework, attains the form:

$$
\phi_{\xi \xi}(\xi)-\alpha \phi(\xi)+\gamma \phi^{3}(\xi)=0
$$

where $\gamma=\frac{G}{2 R_{0}^{2}\left(J-L_{2}\right)}$ with $G=\frac{2 E_{b}}{1-v^{2} / c^{2}}$, and $\alpha=k^{2}+\frac{L-\hbar \Omega}{R_{0}^{2}\left(J-L_{2}\right)}$ in the case of the symmetric amplitudes, while, for the antisymmetric amplitudes, we have $\gamma=\frac{G}{2 R_{0}^{2}\left(J+L_{2}\right)}, \alpha=k^{2}-\frac{L+\hbar \Omega}{R_{0}^{2}\left(J+L_{2}\right)}$, and $L=L_{1}+2 L_{2}$.

As we mentioned, the pulse shape of the charge envelope function can be expected under the boundary conditions (14). In both cases (symmetric and antisymmetric), the nonlinear differential equation (18) has the soliton solution when $\alpha>0$ and $\gamma>0$. In this case, we have the following soliton solution:

$$
\phi(\xi)=\frac{\phi_{0}}{\cosh (\sqrt{\alpha} \xi)}, \quad \phi_{0}^{2}=2 \alpha / \gamma,
$$

where $\phi_{0}$ is the soliton amplitude, and $\xi=x-v t$. In the case of the symmetric solution we have:

$$
\begin{aligned}
\phi_{0}^{2} & =\frac{G}{16\left(J-L_{2}\right)}, \\
\alpha & =\frac{\gamma}{2} \cdot \phi_{0}^{2}=\frac{G^{2}}{64 R_{0}^{2}\left(J-L_{2}\right)^{2}}, \\
\hbar \Omega & =\frac{\hbar^{2} v^{2}}{4 R_{0}^{2}\left(J-L_{2}\right)}-\frac{G^{2}}{64\left(J-L_{2}\right)}+L,
\end{aligned}
$$


while in the antisymmetric case, we obtain:

$$
\begin{aligned}
\phi_{0}^{2} & =\frac{G}{16\left(J+L_{2}\right)}, \\
\alpha & =\frac{\gamma}{2} \cdot \phi_{0}^{2}=\frac{G^{2}}{64 R_{0}^{2}\left(J+L_{2}\right)^{2}}, \\
\hbar \Omega & =\frac{\hbar^{2} v^{2}}{4 R_{0}^{2}\left(J+L_{2}\right)}-\frac{G^{2}}{64\left(J+L_{2}\right)}-L .
\end{aligned}
$$

Bearing in mind the definition of $\gamma$ in (18), for $J>0, J \gg\left|L_{1,2}\right|$, one can conclude that $v<c$ ("subsonic" soliton solution). It should be noted that the state of the considered system is determined by the set of parameters $\left(E_{b}\right.$, $J_{1}, L_{1}, L_{2}, J_{\delta}$ ). These parameters depend on the physical properties of the macromolecule under consideration. In fact, the soliton's velocity should be added to the parameters that determine the state of the system. This parameter is "external" in the sense that it does not depend on the physical properties of the macromolecule itself, but it is determined either by the environment (for example, by the fact that the ensemble of induced charges is in thermal equilibrium with the environment in which the macromolecule is placed), or by some other way (during a chemical reaction, during an experiment, etc.).

\section{Energy spectra for soliton}

\subsection{Energy spectra of linear excitations}

Let us now examine the energetic stability of the soliton solution. For this purpose, we have to find the soliton energies for all obtained solutions and compare them with the energy of the quasi-free exciton (charge) state, i.e. the energy corresponding to the functional $\mathcal{H}$, defined by (8), but in the absence of the exciton-phonon coupling. Let us first find the energy of free exciton states: setting $F_{q}=0$, taking the exciton amplitudes in the form $\psi_{j}(x, t)=$ $A_{j} \cdot \mathrm{e}^{-\frac{i}{\hbar} \mathcal{E}(k) \cdot t+i k x}$ and put them into equation of motion for the exciton amplitude (9), we obtain the linear system of equations for amplitudes $A_{1}$ and $A_{2}$ in the form:

$$
\begin{aligned}
& {\left[\mathcal{E}(k)-\mathcal{E}_{0}+2 J_{1}\left(1-\frac{R_{0}^{2} k^{2}}{2}\right)-2 J_{\delta}\left(1-\frac{\delta^{2} R_{0}^{2} k^{2}}{2}\right)\right] \cdot A_{j}} \\
& -\left[L_{1}+2 L_{2}\left(1-\frac{R_{0}^{2} k^{2}}{2}\right)\right] \cdot A_{3-j}=0 .
\end{aligned}
$$

It should be noted that $k$ appearing in (22) is the wave vector of free quasiparticle in a periodic structure with the spatial period $R_{0}$, and, consequently, $k \in\left[-\pi / R_{0}, \pi / R_{0}\right]$. This parameter should not be confused with the parameter of the same notation, defined by the relation (17), which represents the wave vector of the soliton carrier wave. The obtained system of equations has nontrivial solutions provided that the determinant of the system is equal to zero. From this condition, we find that the energy spectrum for quasi-free excitons has two bands:

$$
\begin{aligned}
\mathcal{E}_{A, S}^{\text {free }}(k) & =\mathcal{E}_{0}-2 J_{1}\left(1-\frac{R_{0}^{2} k^{2}}{2}\right)+2 J_{\delta}\left(1-\frac{\delta^{2} R_{0}^{2} k^{2}}{2}\right) \\
& \mp\left[L_{1}+2 L_{2}\left(1-\frac{R_{0}^{2} k^{2}}{2}\right)\right] .
\end{aligned}
$$

The sign "-" in (23) corresponds to the antisymmetric solution of (22) (denoted by $A$ ), while the sign " +" corresponds to the symmetric solution of (22) (denoted by $S$ ). We can see that the spectrum of the linear excitations splits into two bands: symmetric and antisymmetric ones, separated by the energy gap. The width of this gap depends on the transfer integrals $L_{1}$ and $L_{2}$ only. The corresponding eigenstates are the linear superpositions of the exciton states belonging to different strands. As a consequence, the quasi-free exciton states are hybrid states, fully delocalized, in which the particle probability density is uniformly distributed over both strands.

It is useful to express all energies in term of parameters $S=E_{b} / \hbar \omega_{0}$ and $B=2 J / \hbar \omega_{0}$. The bottom of the energy band of a quasi-free exciton, in the case of the exciton symmetric (antisymmetric) state (since $\left|L_{2}\right|<J_{1}$ ) is:

$$
\begin{aligned}
& \overline{\mathcal{E}}_{A}^{\text {free }}=\frac{\mathcal{E}_{0}}{\hbar \omega_{0}}-B\left(1-p_{\delta}+p_{1}+2 p_{2}\right), \\
& \overline{\mathcal{E}}_{S}^{\text {free }}=\frac{\mathcal{E}_{0}}{\hbar \omega_{0}}-B\left(1-p_{\delta}-p_{1}-2 p_{2}\right) .
\end{aligned}
$$

Here, $\overline{\mathcal{E}}_{A, S}^{\text {free }}=\mathcal{E}_{A, S}^{\text {free }} / \hbar \omega_{0}$ is the bottom of the energy of the antisymmetric (symmetric) quasi-free exciton state, normalized by the characteristic phonon energy $\hbar \omega_{0}$. 


\subsection{Energy spectra of soliton}

The mean value of the exciton Hamiltonian (2) in the continuum approximation is:

$$
\begin{aligned}
& \mathcal{H}_{e}=\left\langle\psi\left|\hat{H}_{e}\right| \psi\right\rangle=\left(\mathcal{E}_{0}-2 J_{1}+2 J_{\delta}\right) \sum_{j} \int \frac{d x}{R_{0}}\left|\psi_{j}\right|^{2} \\
& -J R_{0}^{2} \sum_{j} \int \frac{d x}{R_{0}}\left\{\psi_{j}^{*} \frac{\partial^{2} \psi_{j}}{\partial x^{2}}\right\}+\left(L_{1}+2 L_{2}\right) \sum_{j} \int \frac{d x}{R_{0}}\left\{\psi_{j}^{*} \psi_{3-j}\right\} \\
& +L_{2} R_{0}^{2} \sum_{j} \int \frac{d x}{R_{0}}\left\{\psi_{j}^{*} \frac{\partial^{2} \psi_{3-j}}{\partial x^{2}}\right\}-\frac{2 E_{b}}{1-v^{2} / c^{2}} \sum_{j} \int \frac{d x}{R_{0}}\left|\psi_{j}\right|^{4} .
\end{aligned}
$$

After substitution (13) into (25), we express the mean value of the exciton Hamiltonian in terms of the soliton amplitudes $A_{j}$ :

$$
\begin{aligned}
& \mathcal{H}_{e}=\left(\mathcal{E}_{0}-2 J_{1}+2 J_{\delta}\right)+\left(J_{1}-\delta^{2} J_{\delta}\right) R_{0}^{2} k^{2} \\
& +\alpha\left(J_{1}-\delta^{2} J_{\delta}\right) R_{0}^{2}-\frac{2}{3} \frac{\phi_{0}^{4} \gamma R_{0}}{\sqrt{\alpha}}\left(J_{1}-\delta^{2} J_{\delta}\right) \\
& +\left[\left(L_{1}+2 L_{2}\right)-L_{2} R_{0}^{2} k^{2}-\alpha L_{2} R_{0}^{2}+\frac{2}{3} \frac{\phi_{0}^{4} \gamma R_{0}}{\sqrt{\alpha}} L_{2}\right] \cdot \sum_{j} A_{j} A_{3-j} \\
& -\frac{4 G}{3} \frac{\phi_{0}^{4} \gamma R_{0}}{\sqrt{\alpha}} \sum_{j} A_{j}^{4} .
\end{aligned}
$$

To obtain the above equation, we used the results of the following integrals:

$$
I_{1}=\int_{-\infty}^{+\infty} \frac{d \xi}{R_{0}} \phi^{2}(\xi)=1
$$

(as a consequence of the normalization conditions),

$$
I_{2}=\int_{-\infty}^{+\infty} \frac{d \xi}{R_{0}} \phi(\xi) \phi_{\xi}(\xi)=0, \quad I_{3}=\int_{-\infty}^{+\infty} \frac{d \xi}{R_{0}} \phi^{4}(\xi)=\frac{4}{3} \cdot \frac{\phi_{0}^{4}}{R_{0} \sqrt{\alpha}}, \quad I_{4}=\int_{-\infty}^{+\infty} \frac{d \xi}{R_{0}} \phi(\xi) \phi_{\xi \xi}(\xi)=\frac{2}{3} \cdot \frac{\phi_{0}^{4} \gamma}{R_{0} \sqrt{\alpha}}-\alpha
$$

In the case of the antisymmetric solution, the soliton energy expressed in terms of the set of the basic system parameters, normalized to the energy $\hbar \omega_{0}$, in the "subsonic" limit $(v / c \ll 1)$, is:

$$
\begin{aligned}
\overline{\mathcal{E}}_{A}^{\text {sol }} & =\left\{\frac{\mathcal{E}_{0}}{\hbar \omega_{0}}-\frac{B}{2}\left(2+p_{1}+2 p_{2}\right)+\frac{S^{2}}{24 B} \cdot \frac{1}{1+p_{2}}-\frac{S^{3}}{3 B^{2}} \cdot \frac{1}{\left(1+p_{2}\right)^{2}}\right\} \\
& +\frac{v^{2}}{c^{2}}\left\{\frac{1}{8 B} \cdot \frac{1}{1+p_{2}}+\frac{S^{2}}{12 B} \cdot \frac{1}{1+p_{2}}-\frac{S^{3}}{B^{2}} \cdot \frac{1}{\left(1+p_{2}\right)^{2}}\right\} .
\end{aligned}
$$

At the same time, the normalized energy of the symmetric soliton is:

$$
\begin{aligned}
\overline{\mathcal{E}}_{S}^{\text {sol }} & =\left\{\frac{\mathcal{E}_{0}}{\hbar \omega_{0}}-\frac{B}{2}\left(2-p_{1}-2 p_{2}\right)+\frac{S^{2}}{24 B} \cdot \frac{1}{1-p_{2}}-\frac{S^{3}}{3 B^{2}} \cdot \frac{1}{\left(1-p_{2}\right)^{2}}\right\} \\
& +\frac{v^{2}}{c^{2}}\left\{\frac{1}{8 B} \cdot \frac{1}{1-p_{2}}+\frac{S^{2}}{12 B} \cdot \frac{1}{1-p_{2}}-\frac{S^{3}}{B^{2}} \cdot \frac{1}{\left(1-p_{2}\right)^{2}}\right\} .
\end{aligned}
$$

The obtained relations (27) and (28) make it possible to analyze the energy stability of soliton solutions and, consequently, represent the basic result of the presented analysis. Comparing them with the corresponding energies of quasi-free excitons (24), one can conclude whether the formation of a soliton is more favorable in comparison with the state of a quasi-free charge from the energy point of view. For our purpose, it is important to investigate the values of the system parameters that provide the condition $E^{S o l}<E^{\text {free }}$ in the region where $B>S$. The results are shown in Figs. 2 and 3. The left panels of the figures demonstrate that the stable soliton solutions of extra charge can be formed in DNA-like structures with small values of $\left|p_{1}\right|$, i.e. $\left|L_{1}\right| \ll|J|$, and in which the adiabatic parameter $B$ is not very much higher than the coupling constant $S$. Moreover, it can be seen that antisymmetric solitons are more favorable when the transfer integrals of the same strand $J$ and different chains $L_{1,2}$ have the same signs $\left(p_{1,2}>0\right)$, while symmetric ones might be realized when their signs are different $\left(p_{1,2}<0\right)$. 

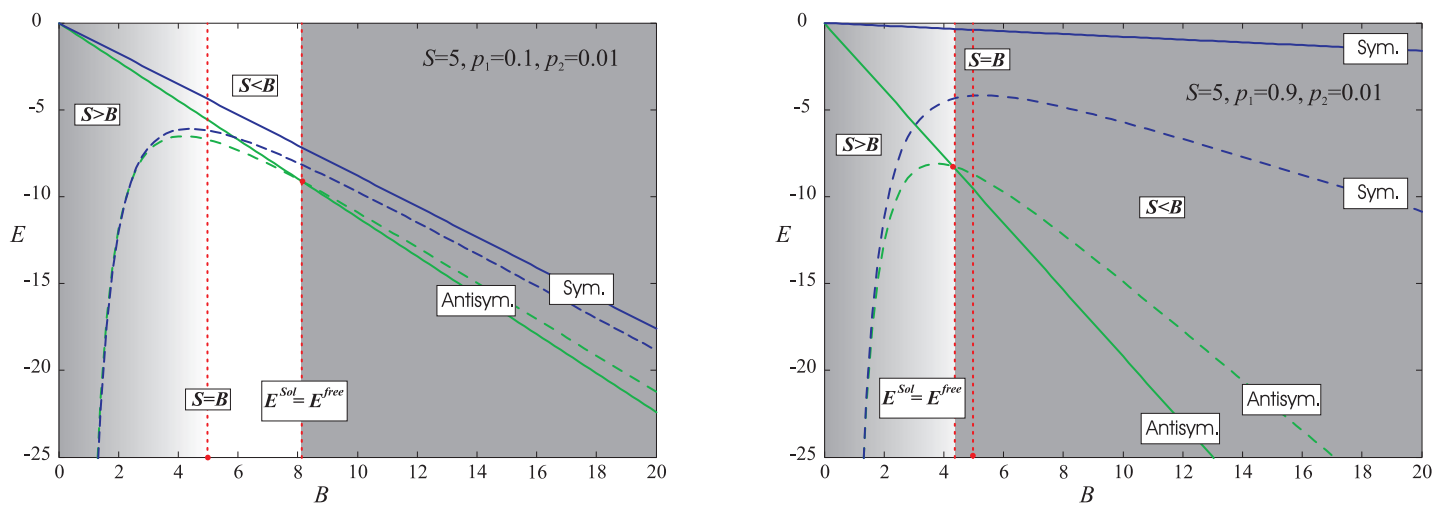

FIG. 2. The dependence of the soliton energy on the adiabatic parameter $B$ for $S=5, p_{\delta}=0$, $v / c=0$, and $p_{1}=0.1, p_{2}=0.01$ (left panel), $p_{1}=0.9, p_{2}=0.01$ (right panel). Full lines correspond to the energy of the free extra charge, while the dashed ones correspond to the energy of the soliton.
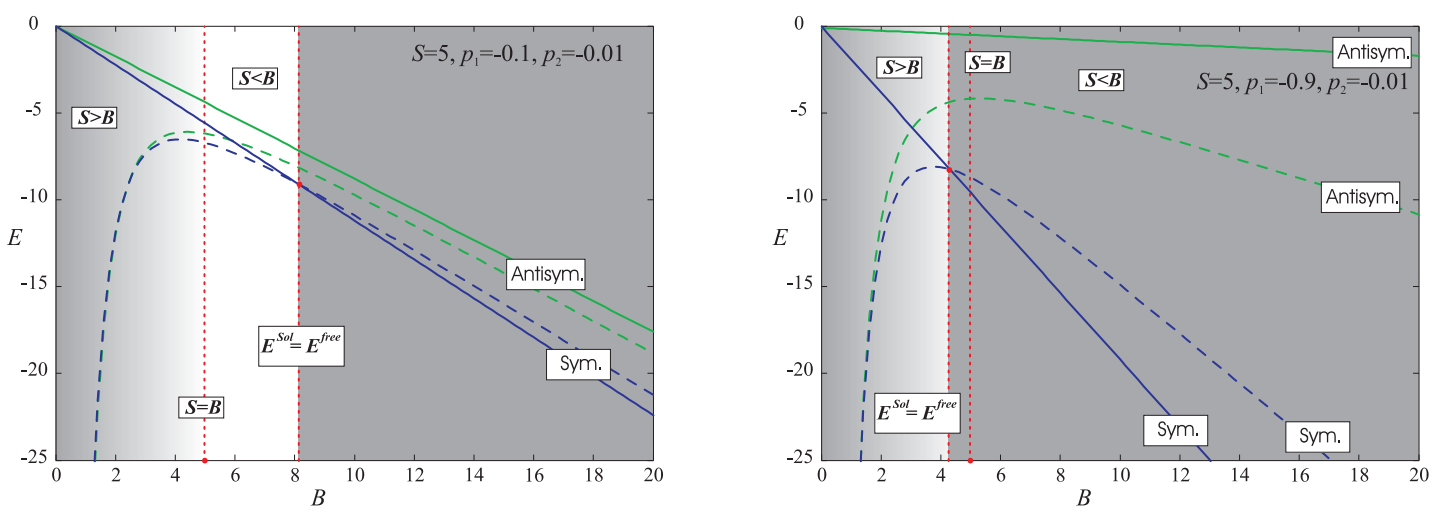

FIG. 3. The same as in Fig. 2 with $p_{1}=-0.1, p_{2}=-0.01$ on the left panel, and $p_{1}=-0.9$, $p_{2}=-0.01$ on the right panel.

\section{Conclusion}

In this paper, the possibility of stable long-distance migration of charge carriers in DNA-like macromolecular structures in the form of an adiabatic soliton was investigated. We derived the conditions for the formation of two types of soliton solutions: symmetric and antisymmetric. Analysis of the soliton energy spectra showed that when the transfer integrals of the same strand $J$ and different chains $L_{1,2}$ have the same signs $\left(p_{1,2}>0\right)$, then it is more favorable for antisymmetric solitons, while the symmetric ones might be realized when signs of the transfer integrals are different $\left(p_{1,2}<0\right)$. It should also be noted that the formation of stable soliton solutions of both types can occur in structures only with small values of $\left|p_{1}\right|$, i.e. $\left|L_{1}\right| \ll|J|$, and in which the adiabatic parameter $B$ is not very much higher than the coupling constant $S$. In addition, according to the presented model, the helical structure of DNA-like macromolecules does not have a large effect on the formation of solitons because of the large helix pitch. This only affects the effective decrease in the overlap integral $J_{1}$, i.e. to the parameter $B$.

\section{Acknowledgements}

This work is partly supported by the Serbian Ministry of Education and Science under contracts Nos. III-45010 and OI-171009, the COST action No. 17139, and the Project within the Cooperation Agreement between the JINR, Dubna, Russian Federation, and Ministry of Education and Science of Republic of Serbia.

\section{References}

[1] Dekker C., Ratner M.A. Electronic properties of DNA. Phys. World, 2001, 14 (8), P. 29-33.

[2] Rakovic D. Osnovi biofizike. IASC\&IEFPG, Beograd, 2008, 368 p. (in Serbian).

[3] Giese B. Long-Distance Charge Transport in DNA: The Hopping Mechanism. Acc. Chem. Res., 2000, 33 (9), P. $631-636$. 
[4] Schuster G.B. Long-Range Charge Transfer in DNA: Transient Structural Distortions Control the Distance Dependence. Acc. Chem. Res., 2000,33 (4), P. 253-260.

[5] Heller A. Spiers Memorial Lecture. On the hypothesis of cathodic protection of genes. Faraday Discuss., 2000,116, P. 1-13.

[6] Davydov A.S. Solitons in molecular systems. Phys. Scr., 1979, 20 (2), P. 387-394.

[7] Cevizovic D., Ivic Z., Przulj Z., Tekic J., Kapor D. Interchain coupling effects on large acoustic polaron in two parallel molecular chains. Chem. Phys., 2013, 426, P. 9-15.

[8] Giese B. Electron transfer in DNA. Curr. Opin. Chem. Biol., 2002, 6 (5), P. 612-618.

[9] Hawke L.G.D., Kalosakas G., Simserides C. Electronic parameters for charge transfer along DNA. Eur. Phys. J. E, 2010, 32 (3), P. $291-306$.

[10] Holstein T. Studies of polaron motion: Part I. The molecular-crystal model. Annals of Physics, 1959, 8 (3), P. $325-342$.

[11] Cevizovic D., Ivic Z., Galovic S., Reshetnyak A., Chizhov A. On the vibron nature in the system of two parallel macromolecular chains: The influence of interchain coupling. Phys. B, 2016, 490, P. 9-15.

[12] Rashba E.I. Self-trapping of excitons. Excitons, North-Holland, Amsterdam: 1982. P. 543-602.

[13] Brown D.W., Ivic Z. Unification of polaron and soliton theories of exciton transport. Phys. Rev. B, 1989,40 (14), P. 9876-9887.

[14] Emin D. Self-trapping in quasi-one-dimensional solids. Phys. Rev. B, 1986, 33 (6), P. 3973-3975.

[15] Yarkony D., Silbey R. Variational approach to exciton transport in molecular crystals. J. Chem. Phys., 1977,67 (12), P. $5818-5827$.

[16] Lang I.G., Firsov Yu.A. Kinetic theory of semiconductors with low mobility. Soviet Physics JETP, 1963, 16 (5), P. 1301-1312.

[17] Maniadis P., Kalosakas G., Rasmussen K.O., Bishop A.R. Polaron normal modes in the Peyrard-Bishop-Holstein model. Phys. Rev. B, 2003, 68 (17), 174304 (11 p).

[18] Kalosakas G. Charge transport in DNA: Dependence of diffusion coefficient on temperature and electron-phonon coupling constant. Phys. Rev. E, 2011, 84 (5), 051905 (6 p).

[19] Baeriswyl D., Maki K. Interchain order, soliton confinement, and electron-hole photogeneration in trans-polyacetylene. Phys. Rev. B, 1988, 38 (12), P. 8135-8141.

[20] Davydov A.S. Biologya i Kvantovaya Mehanika. Naukova Dumka, Kiev, 1979. P. 142-216. (In Russian)

[21] Davydov A.S. The theory of contraction of proteins under their excitation. J. Theor. Biol., 1973, 38 (3), P. $559-569$.

[22] Emin D. On the existence of free and self-trapped carriers in insulators: an abrupt temperature-dependent conductivity transition. Adv. Phys., 1973, 22 (1), P. 57-116.

[23] Pekar S.I. Issledovaniya po elektronnoi teorii kristaslov. Gostekhizdat, Moskva, 1951. 256 p. (in Russian).

[24] Pekar S.I. Autolocalization of the electron in an inertially polarizable dielectric medium. Zh. Eksp. Teor. Fiz., 1946, 16, P. $335-343$.

[25] Zdravkovic S., Sataric M. Parameter selection in a Peyrard-Bishop-Dauxois model for DNA dynamics. Phys. Lett. A, 2009, 373 (31), P. 27392745 .

[26] Kitoh-Nishioka H., Ando K. Charge-Transfer Matrix Elements by FMO-LCMO Approach: Hole Transfer in DNA with Parameter Tuned Range-Separated DFT. Chem. Phys. Lett., 2015, 621, P. 96-101.

[27] Dauxois T., Peyrard M. Physics of Solitons. Cambridge University Press, Cambridge, 2004. $436 \mathrm{p}$.

[28] Firsov Yu.A. Polarons. Ed. Yu. A. Firsov, Nauka, Moskva, 1975. 423 p. (in Russian).

[29] Wang X., Brown D.W., Lindberg K., West B. Alternative formulation of Davydov theory of energy transport in biomolecular systems. Phys. Rev. A, 1988, 37 (9), P. 3557-3566.

[30] Ivic Z., Zekovic S., Przulj Z. Radiative decay of the one-dimensional large acoustic polaron. Phys. Lett. A, 2002 , 306 (2-3), P. $144-152$.

[31] Kevrekidis P.G., Frantzeskakis D.J., Carretero-Gonziez R. (Eds.) Emergent nonlinear phenomena in Bose-Einstein condensates. Springer, Berlin, 2008. 405 p.

[32] Kivshar Yu.S., Agrawal G.P. Optical Solitons: From Fibers to Photonic crystals. Academic Press, New York: 2003.540 p.

[33] Shi X., Malomed B.A., Ye F., Chen X. Symmetric and asymmetric solitons in a nonlocal nonlinear coupler. Phys. Rev. A, 2012, 85 (5), 053839 (8 p).

[34] Akhmediev N.N., Ostrovskaya E.A. Elliptically polarized spatial solitons in cubic gyrotropic materials. Opt. Commun., 1996, 132 (1-2), P. 190-204. 